\title{
Survey of experimental results in high-contrast imaging for future exoplanet missions
}

P. R. Lawson, R. Belikov, W. Cash, M. Clampin, T. Glassman, et al.

P. R. Lawson, R. Belikov, W. Cash, M. Clampin, T. Glassman, O. Guyon, N. J. Kasdin, B. D. Kern, R. Lyon, D. Mawet, D. Moody, R. Samuele, E. Serabyn, D. Sirbu, J. Trauger, "Survey of experimental results in highcontrast imaging for future exoplanet missions," Proc. SPIE 8864, Techniques and Instrumentation for Detection of Exoplanets VI, 88641F (26 September 2013); doi: 10.1117/12.2021302

EviE Event: SPIE Optical Engineering + Applications, 2013, San Diego, California, United States 


\title{
Survey of experimental results in high-contrast imaging for future exoplanet missions
}

\author{
P. R. Lawson ${ }^{1}$, R. Belikov², W. Cash ${ }^{3}$, M. Clampin 4 , T. Glassman ${ }^{5}$, O. Guyon ${ }^{6}$, \\ N. J. Kasdin ${ }^{7}$, B. D. Kern ${ }^{1}$, R. Lyon ${ }^{4}$, D. Mawet ${ }^{8}$, D. Moody ${ }^{1}$, R. Samuele ${ }^{5}$, \\ E. Serabyn ${ }^{1}$, D. Sirbu ${ }^{7}$, and J. Trauger ${ }^{1}$ \\ ${ }^{1}$ Jet Propulsion Laboratory, Caltech, 4800 Oak Grove Drive, Pasadena, CA 91109; \\ ${ }^{2}$ NASA Ames Research Center, Space Science and Astrobiology, Moffett Field, CA 94035; \\ ${ }^{3}$ University of Colorado, CASA, Campus Box 389, Boulder, CO 80309; \\ 4NASA Goddard Space Flight Center, Code 667, Greenbelt, MD 20771; \\ ${ }^{5}$ Northrop Grumman Aerospace Systems, Redondo Beach, CA 90278; \\ ${ }^{6}$ University of Arizona, Steward Obs., 933 North Cherry Ave, Tucson, AZ 85721; \\ 7Princeton University, MAE, D207 Engineering Quad, Princeton, NJ 08544 \\ ${ }^{8}$ European Southern Observatory, Alonso de Cordova 3107, Vitacura, Santiago, Chile
}

\begin{abstract}
We present and compare experimental results in high contrast imaging representing the state of the art in coronagraph and starshade technology. These experiments have been undertaken with the goal of demonstrating the capability of detecting Earth-like planets around nearby Sun-like stars. The contrast of an Earth seen in reflected light around a Sun-like star would be about $1.2 \times 10^{-10}$. Several of the current candidate technologies now yield raw contrasts of $1.0 \times 10^{-9}$ or better, and so should enable the detection of Earths, assuming a gain in sensitivity in post-processing of a factor of 10 . We present results of coronagraph and starshade experiments conducted at visible and infrared wavelengths. Cross-sections of dark fields are directly compared as a function of field angle and bandwidth. The strength and differences of the techniques are compared.
\end{abstract}

Keywords: Exoplanets, coronagraphs, occulters, starshades, starlight suppression

\section{INTRODUCTION}

This paper reviews the current state-of-the-art of starlight suppression for exoplanet detection at visible wavelengths. NASA's Exoplanet Exploration Program has promoted research in this field with the aim of enabling a future mission that could detect and measure the spectra of Earth-like planets around nearby stars. In prior years progress in this field has been reported independently by the researchers involved. For this paper the experimental data was gathered from each researcher and was analyzed uniformally to illustrate the achieved contrast as a function of angular separation. The results that are plotted and tabulated in this paper are consistent with results reported earlier, but yet provide more detail and insight into the relative progress of each technique.

The key instrument performance requirement that drives the technology effort is the ability to suppress starlight to a level where the detection of Earth-like planets becomes possible. To detect a sample of Earth-like planets an observatory must be capable of detecting exoplanets up to 26 magnitudes fainter than their host stars, implying a performance level enabling contrasts of better than $10^{-10}$ at visible wavelengths. In the figures in this paper, contrasts are demonstrated at this level in narrow-band light and near $10^{-9}$ at broader bandwdiths.

Further author information: (Send correspondence to P.R.L.)

P.R.L.: E-mail: Peter.R.Lawson@jpl.nasa.gov, Telephone: 18183540747

Techniques and Instrumentation for Detection of Exoplanets VI, edited by Stuart Shaklan, Proc. of SPIE Vol. 8864, 88641F · @ 2013 SPIE · CCC code: 0277-786X/13/\$18 · doi: 10.1117/12.2021302 


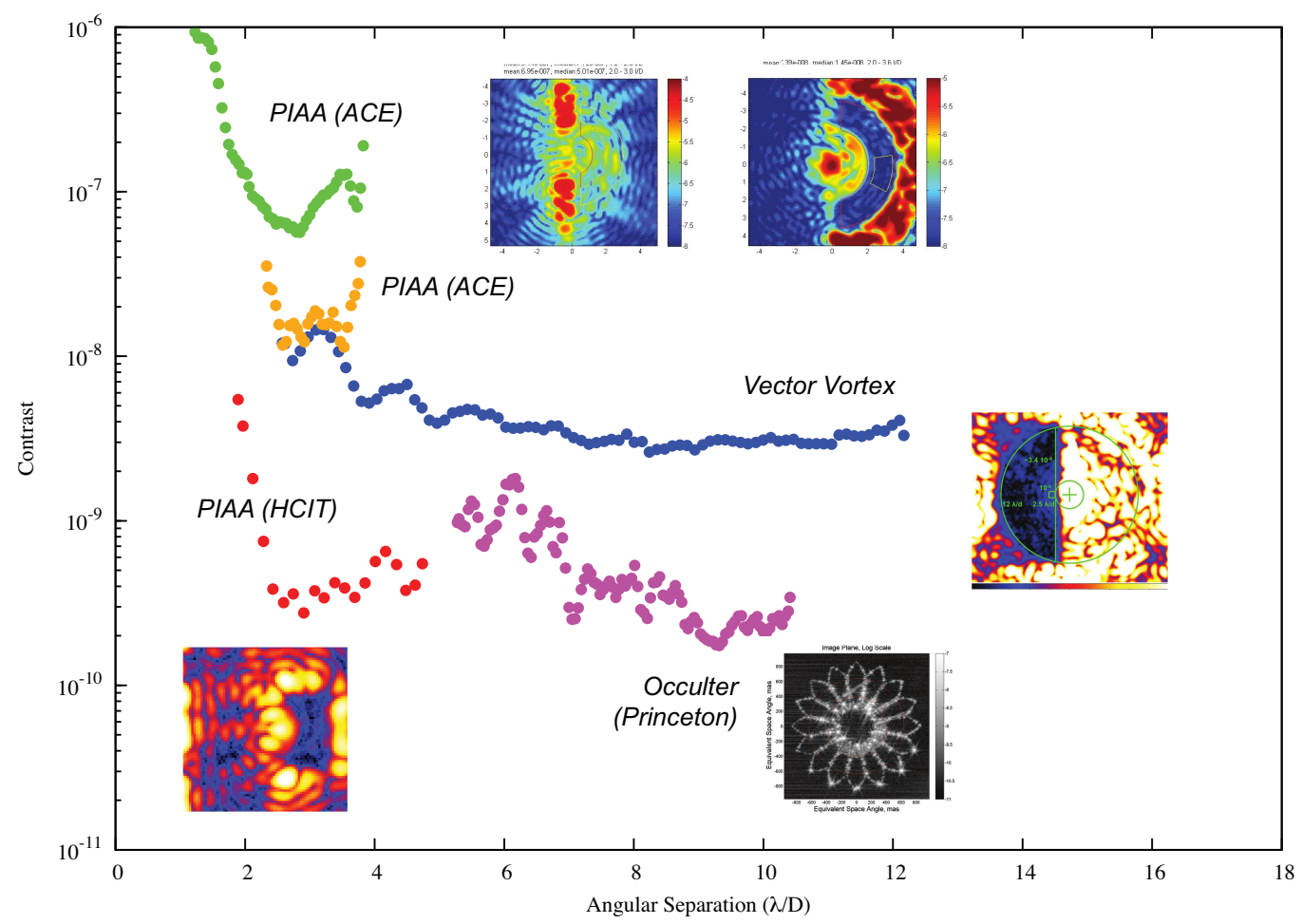

Figure 1: Coronagraph and starshade laboratory demonstrations using laser light (monochromatic).

\section{CORONAGRAPH STARLIGHT SUPPRESSION}

For this survey, the original data files were obtained from each Principal Investigator and were reprocessed to yield estimates of the contrast versus angular separation. The bounds defining the borders of the dark hole (for coronagraphs) were adjusted so that the reported averages were not biased by bright features at the edges of the dark hole. The reported contrasts are averages approximately two pixels in width and averaged in arcs equidistant from the simulated star (azimuthal averages). The averages reported here correspond well to the averages reported by the original authors themselves, but now show how the contrast varies as a function of angular separation, expressed here in $\lambda / D$.

Additional metrics were also calculated, including the mean contrast across the dark hole (for coronagraphs), the variance of the contrast, the variance as a function of angular separation, and the angular extent of the dark hole.

The co-authors felt strongly that the throughput of each method should also be displayed graphically along with the contrast to provide a fair comparison. It was also suggested that the results be reported as $5-\sigma$ contrast levels to be more in accord with the reporting of ground-based results. Time was not available to complete this work. The curves report the raw contrast. The comparison shown here is preliminary and will be elaborated upon for future publication.

\subsection{Hybrid Lyot Masks}

The Hybrid Lyot masks are image-plane masks that appear as a linear fringe pattern of metal deposited on glass, with an additional (thus hybrid) layer of dielectric to compensate for residual phase errors. The detailed designs of the masks are described by Moody et al. (2008) in Ref. 1. The results described here were reported earlier by Trauger et al. (2012), Ref. 2.

One deformable mirror (DM) is used in the experiments, whereas two would be used in flight. The masks that have been used have all been linear masks, because the manufacturing process is simpler. Circularly symmetric masks would no doubt be manufactured for a flight mission. Although the designs of circular masks exist, none 


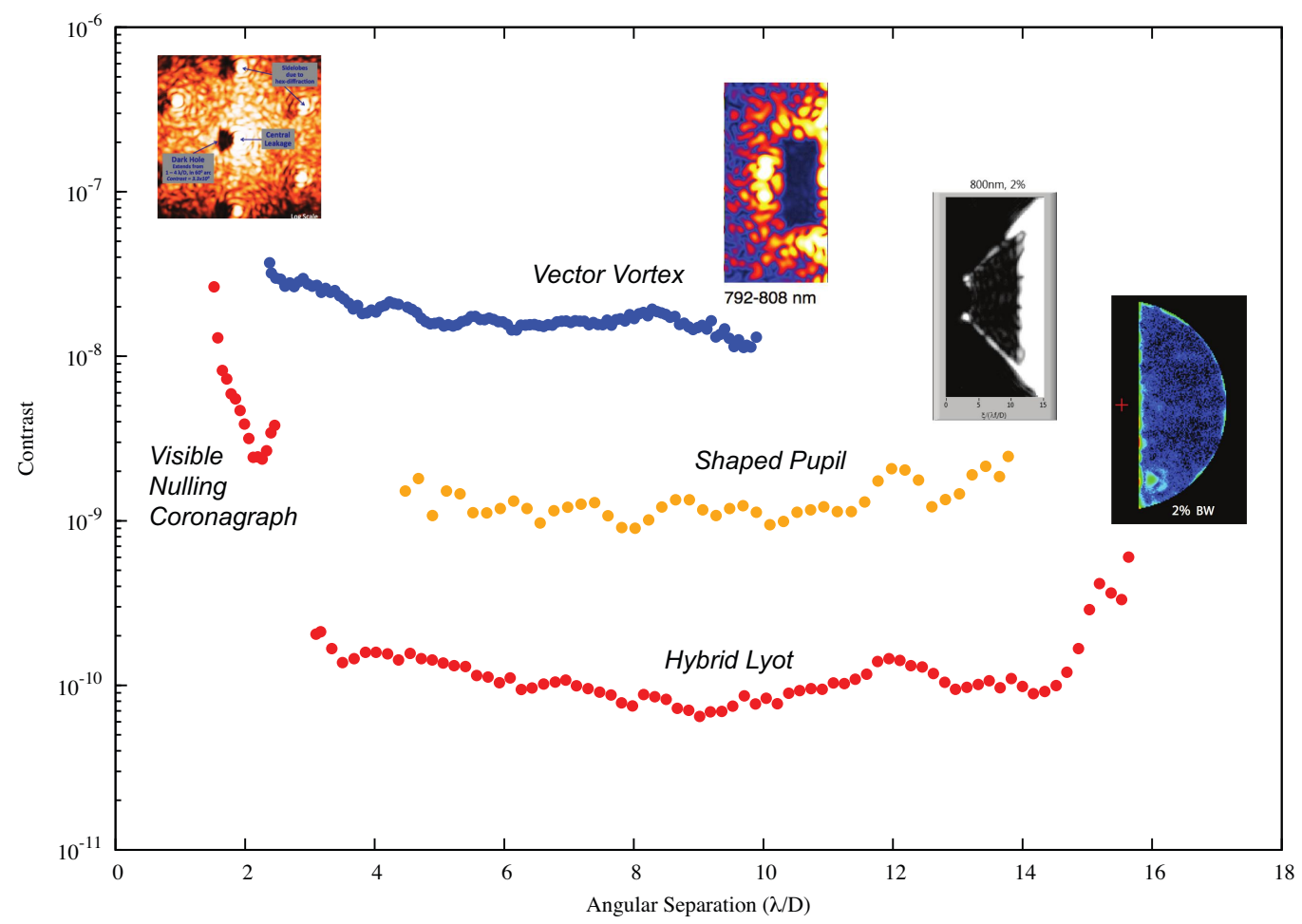

Figure 2: Coronagraph laboratory demonstrations using $2 \%$ bandwidth.

have yet been fabricated. The current limitation in performance is understood to be the ability to accurately deposit the required thickness of dielectric.

The throughput can be estimated from the effective pupil shear used in the mask design, which for the results reported here was a shear of $36 \%$ of the pupil thus yielding a throughput of $56 \%$ past the Lyot stop. Polarizers are not used.

\subsection{Phase-Induced Amplitude Apodization}

Phase-Induced Amplitude Apodization (PIAA) uses pairs of aspheric mirrors to reshape the pupil-plane intensity distribution, providing a Gaussian-like (prolate spheroidal) distribution and eliminating diffraction sidelobes. A perspective of future work in this approach is provided by Guyon in Ref. 3. The results reported here are from two separate facilities. In-air experiments are reported from the NASA Ames Coronagraph Experiment (ACE) by Belikov et al. (Refs. 4, and 5), and from the vacuum High Contrast Imaging Testbed (HCIT) at the Jet Propulsion Laboratory, Ref. 6.

For the flight configuration, there would be two DMs located before the input PIAA optics and there would be a second (inverse) set of PIAA optics after the focal-plane mask and prior to the science camera. With this arrangement the outer working angle is limited by the number of actuators on the DM, as is the case with other coronagraphs. However, for the laboratory demonstrations there is only one DM located between the input PIAA optics and focal-plane mask and no inverse PIAA optics. Having the DM located after the PIAA optics is a convenience that simplifies the wavefront control, because the mapping of the DM elements is not altered by the PIAA optics. The angular extent of the dark holes that are demonstrated in the lab are limited by the number of actuators on the DM and the mapping of the PIAA. The mirror pairs used in the HCIT were not optimized for operations at broad-bandwidths, and new mirrors would be manufactured for flight. Polarizers were used in these experiments to improve the measured contrast. The challenge of implementing PIAA optics lies in the design and manufacturing of these aspheres. 


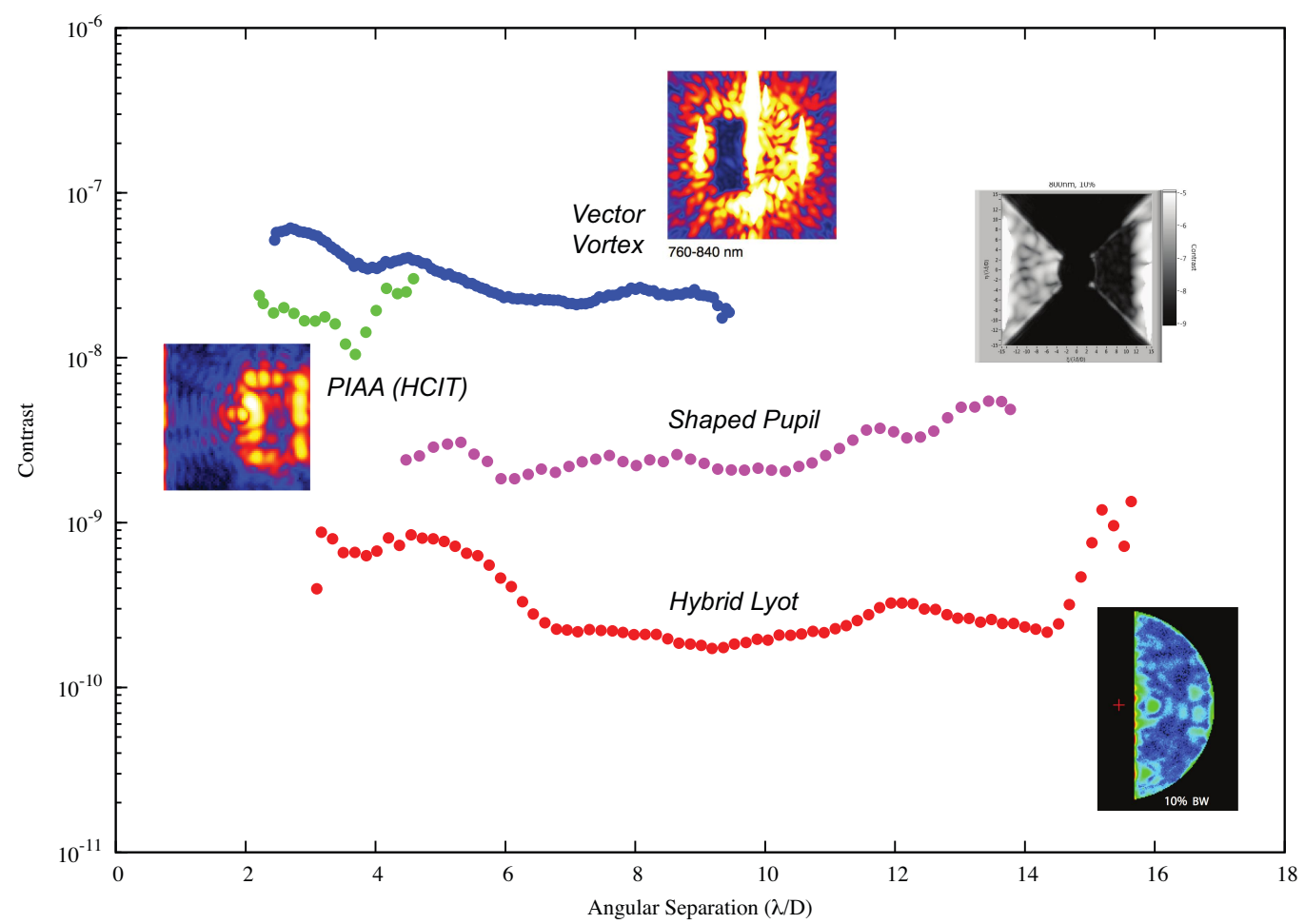

Figure 3: Coronagraph laboratory demonstrations using a 10\% bandwidth.

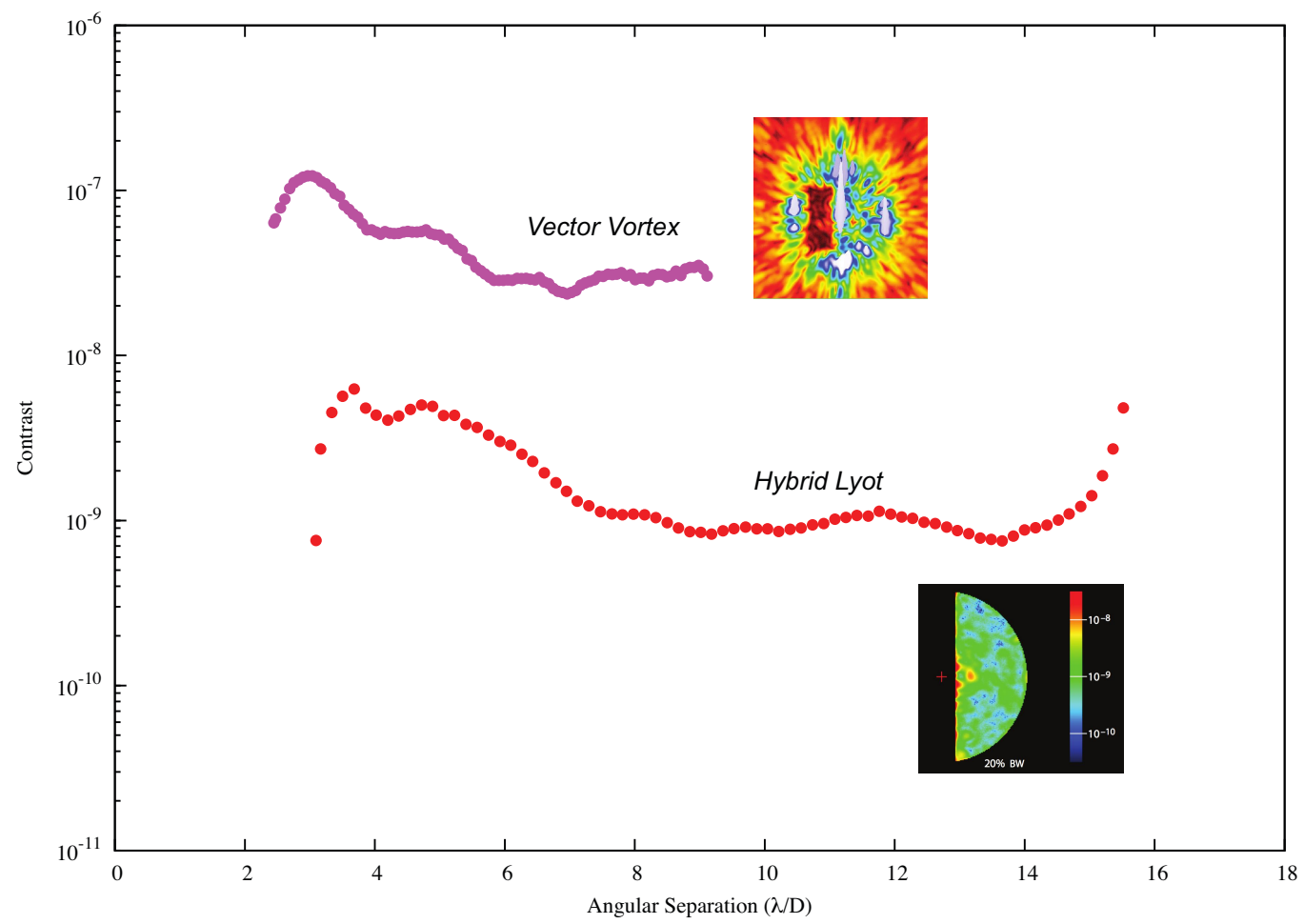

Figure 4: Coronagraph laboratory demonstrations using a $20 \%$ bandwidth. 


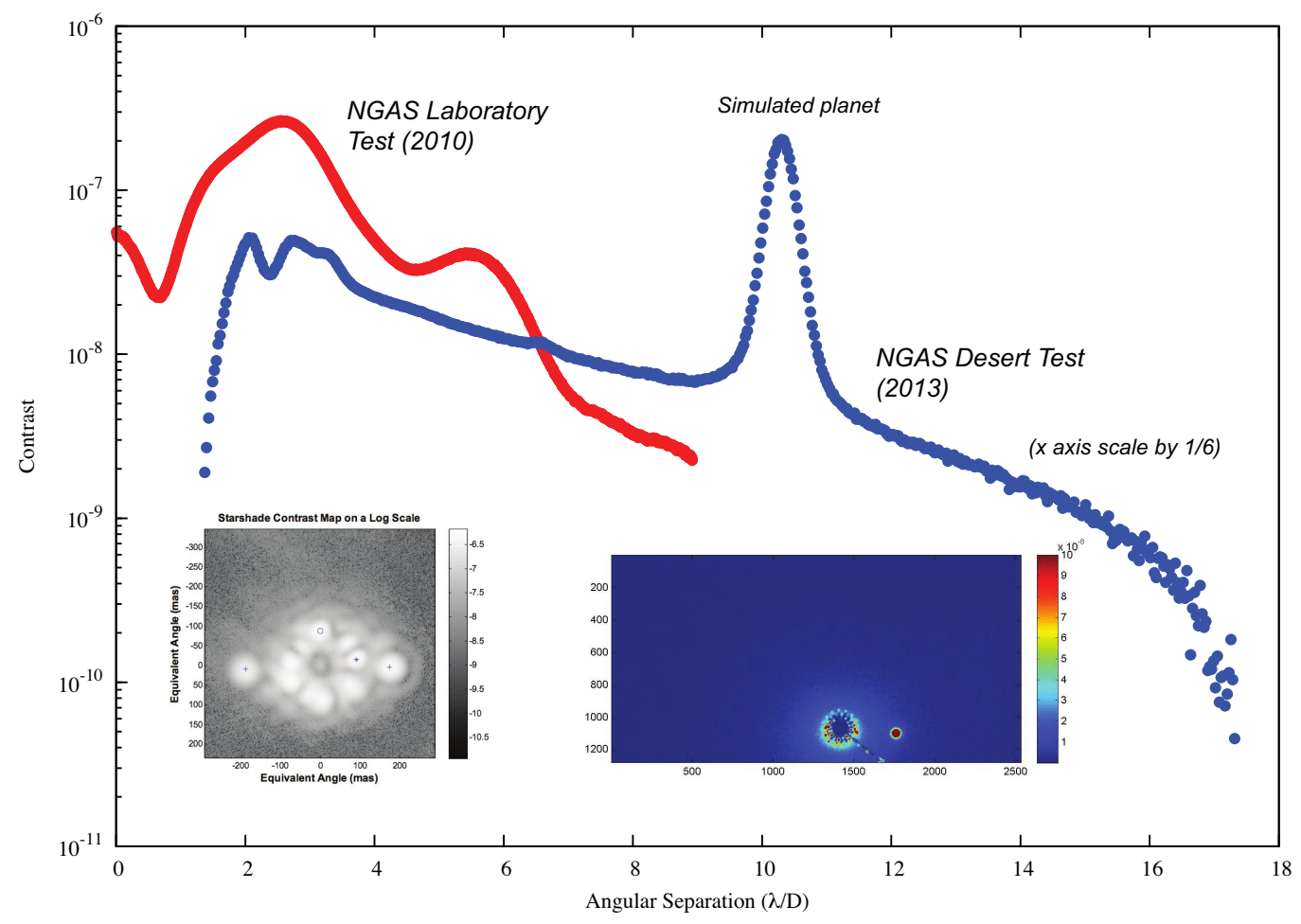

Figure 5: Starshade demonstrations in the lab and in the desert with $50 \%$ bandwidth.

The PIAA optics would ideally have a throughput of $100 \%$, however pupil and image-plane stops reduce the effective throughput to $92 \%$. Because polarizers were used in these experiments, the actual throughput was approximately $46 \%$.

\subsection{Shaped Pupil Masks}

The Shaped Pupil Coronagraph (SPC) is a binary pupil-plane mask that blocks or passes light in different regions of the pupil and thus shapes the point-spread function of the coronagraph in the image plane, digging out one or more dark holes. The results reported here were previously presented by Belikov et al. in Ref. 7 .

These results made use of a linear "Ripple 3" SPC with one deformable mirror and a layout closely similar to that of the Hybrid Lyot and Vector Vortex coronagraphs. It is likely that for a flight mission a new design would be created to take advantage of possible $2 \mathrm{D}$ optimization of the mask. ${ }^{8}$ Two deformable mirrors would be used in flight. The challenge of manufacturing shaped pupil masks lies in the ability to machine features as small as $10-15 \mu \mathrm{m}$ wide and thinned to a thickness of $50 \mu \mathrm{m}$ or less. Narrower features are approximated by hole patterns. ${ }^{7}$

The Ripple 3 mask has a geometric throughput of approximately $30 \%$. The Airy throughput, which also accounts for light scattered in the wings of the point spread function, reduces the overall throughput to $10 \%$. No polarizers were used in these experiments.

\subsection{Visible Nulling Coronagraphs}

A Visible Nulling Coronagraph (VNC) uses an interferometer back-end to reject starlight via interferometric nulling with a sheared pupil. This approach uses a deformable mirror, typically in combination with an array of single-mode optical fibers. The results reported here were previously presented by Lyon et al. (2012), Ref. 9.

In a flight configuration, two VNC combiners would be used in series (one for each of two orthogonal image axes), whereas the results reported here use only a single VNC combiner. For flight a large outer working angle would be achieved using a deformable mirror with at least 925 segments. The lab demonstrations used 
Table 1: Coronagraph Laboratory Results

\begin{tabular}{|c|c|c|c|c|c|c|c|c|c|c|c|}
\hline Method & $\overline{\overline{\text { Design }}}$ & $\overline{\text { Facility }}$ & $\begin{array}{c}\lambda \\
(\mathrm{nm})\end{array}$ & $\begin{array}{l}\Delta \lambda \\
(\%) \\
\end{array}$ & $\begin{array}{c}\text { Mean Raw } \\
\text { Contrast }\end{array}$ & $\begin{array}{c}r_{\min } \\
(\lambda / D) \\
\end{array}$ & $\begin{array}{c}r_{\max } \\
(\lambda / D)\end{array}$ & $\begin{array}{c}\Delta \phi \\
(\mathrm{deg})\end{array}$ & $\begin{array}{c}\text { Hole } \\
\text { Shape }\end{array}$ & $\begin{array}{c}\text { Field } \\
(\lambda / D)^{2}\end{array}$ & $\begin{array}{c}\text { Throughput } \\
(\%)\end{array}$ \\
\hline BLHL & $4^{t h}$ order & HCIT & 800 & 2 & $1.2 \mathrm{e}-10$ & 3.1 & 15.6 & 180 & $\mathrm{D}$ & 283.8 & 56 \\
\hline BLHL & $4^{t h}$ order & HCIT & 800 & 10 & $3.2 \mathrm{e}-10$ & 3.1 & 15.6 & 180 & $\mathrm{D}$ & 283.8 & 56 \\
\hline BLHL & $4^{t h}$ order & HCIT & 800 & 20 & $1.3 \mathrm{e}-09$ & 3.1 & 15.5 & 180 & $\mathrm{D}$ & 285.6 & 56 \\
\hline PIAA & Prolate & $\mathrm{ACE}$ & 650 & 0 & $4.4 \mathrm{e}-07$ & 1.2 & 2.0 & 140 & Arc & 3.1 & 46 \\
\hline PIAA & Prolate & HCIT-2 & 808 & 0 & $5.7 \mathrm{e}-10$ & 1.9 & 4.7 & 180 & Rect & 12.7 & 46 \\
\hline PIAA & Prolate & HCIT-2 & 800 & 10 & $1.8 \mathrm{e}-08$ & 2.2 & 4.6 & 180 & Rect & 9.9 & 46 \\
\hline SPC & Ripple 3 & HCIT & 800 & 2 & $1.2 \mathrm{e}-09$ & 4.5 & 13.8 & 82 & Wedge & 80.9 & 10 \\
\hline SPC & Ripple 3 & HCIT & 800 & 10 & $2.5 \mathrm{e}-09$ & 4.5 & 13.8 & 82 & Wedge & 80.9 & 10 \\
\hline VNC & $\epsilon=0.25$ & GSFC & 633 & 2 & $5.3 \mathrm{e}-09$ & 1.5 & 2.5 & 28 & Arc & 1.0 & 35 \\
\hline VV & TC4 & HCIT & 785 & 0 & $3.6 \mathrm{e}-09$ & 2.6 & 12.2 & 180 & $\mathrm{D}$ & 173.8 & 36 \\
\hline VV & TC4 & HCIT & 800 & 2 & $1.7 \mathrm{e}-08$ & 2.4 & 9.9 & 180 & Rect. & 65.9 & 43 \\
\hline VV & TC4 & HCIT & 800 & 10 & $2.9 \mathrm{e}-08$ & 2.4 & 9.4 & 180 & Rect. & 59.8 & 43 \\
\hline VV & TC4 & HCIT & 800 & 20 & $4.3 \mathrm{e}-08$ & 2.4 & 9.1 & 180 & Rect. & 55.4 & 43 \\
\hline
\end{tabular}

a 169 segment deformable mirror and produced a wedge-shaped dark hole in the region of $2-5 \lambda / D$. The flight combiners will use arrays of single-mode fibers, with the same number of fibers and the same geometrical arrangement as the segments in the DM, to provide spatial filtering and intensity balancing of light from each segment. These arrays were not used for the demonstrations reported here.

The VNC uses a shear of $25 \%$ of the pupil, which yields a geometric throughput of $69 \%$. A linear polarizer was used for these experiments and so the effective throughput was approximately 35\%. A new approach is anticipated for use in flight to allow both polarizations to be measured simultaneously.

\subsection{Vector Vortex Masks}

The Vector Vortex is an image-plane mask that adjusts the phase of the incoming field, producing a rotational phase ramp of two or more even number of cycles to cancel the on-axis starlight. For the results shown here, a liquid-crystal polymer vector vortex was used. These results were reported previously by Mawet et al. in Ref. 10 . More recent results are presented in these proceedings by Serabyn et al. in Ref. 11.

The optical configuration is essentially identical to that of the Hybrid Lyot architecture, having only one DM for current testing, but likely using two for flight. No fundamental change to the mask design would be needed for flight. The current limitation in performance is related to the ability to manufacture masks with a vortex pattern that is maintained to very small offsets from the center of rotation, and to extend the designs to broadband multi-layer masks. For the current experiments, a polarizer is required prior to the pinhole of the source.

The throughput would ideally be 100\%, but as reported in Table 1 of Ref. 10, the Lyot stop was undersized to $85 \%$ for the monochromatic demonstrations and to $92 \%$ for the broadband demonstrations, yielding a $72 \%$ and $85 \%$ transmission respectively. Additionally a polarizer is used at the source (as mentioned above), and so the effective throughput is approximately $36 \%$ monochromatic and $43 \%$ broadband.

\section{STARSHADE STARLIGHT SUPPRESSION}

The starshade demonstrations reported in this paper were primarily conducted to validate the theoretical models of starshade performance, not to demonstrate contrast as a function of angular separation. The results are given here to show the performance achieved to date and to directly compare them with coronagraph results. Results from three independent tests are presented: (1) Monochromatic tests done at Princeton University, Ref. 12; (2) $50 \%$ bandwidth tests done through a 42.8-m vacuum chamber at Northrup Grumman Aerospace Technologies (NGAS), Ref. 13; and (3) 50\% bandwidth tests conducted by NGAS on the desert floor in Death Valley National Park, Ref. 14. The results from Princeton and the NGAS desert experiments were scaled in angular separation for the illustrations in Fig. 1 and Fig. 5. 


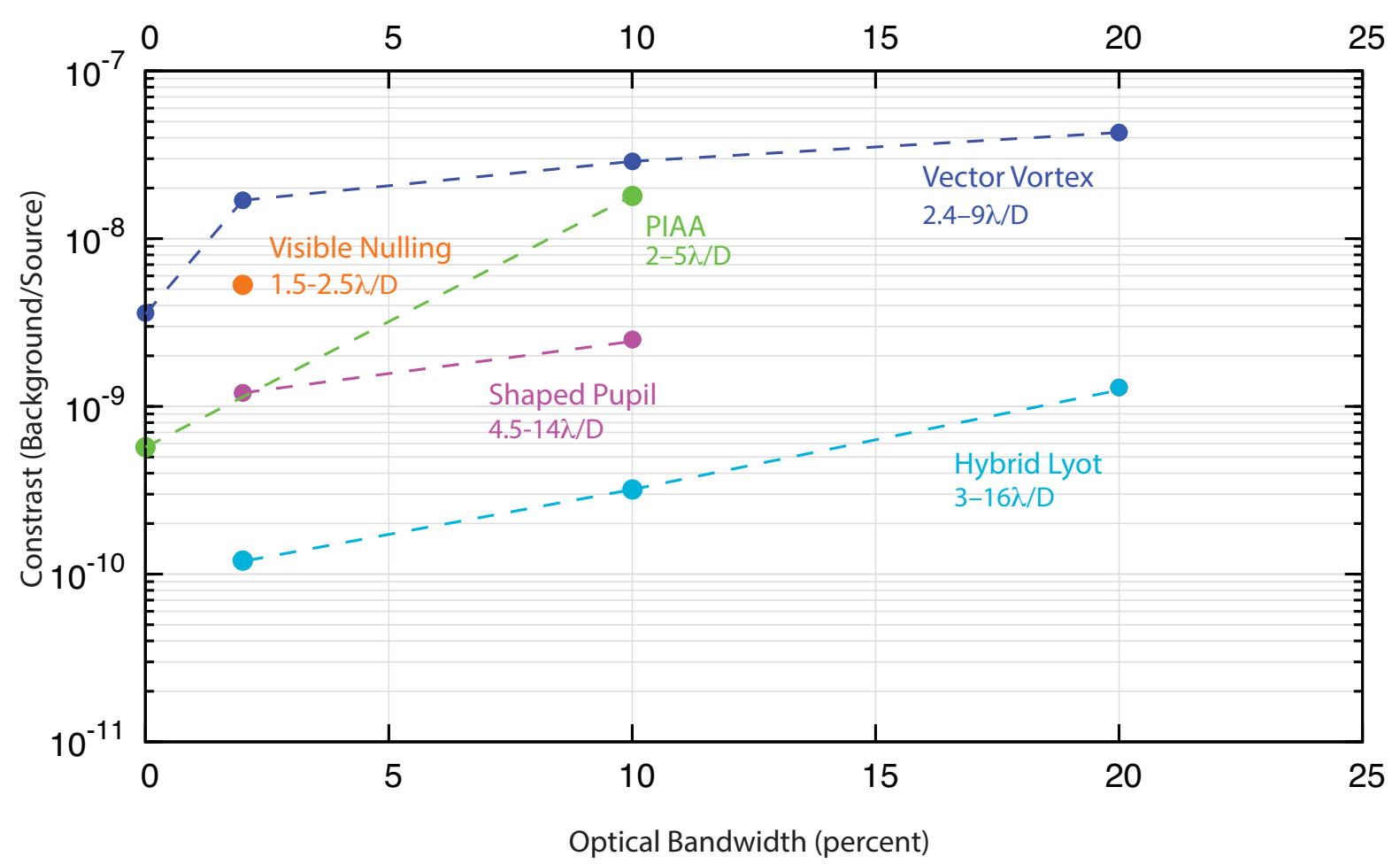

Figure 6: Contrast as a function of bandwidth for coronagraph experiments. The data shown here are extracted directly from Table 1 .

These experiments are designed to measure the starshade's shadow at the same Fresnel number as would be used in an actual flight-system. The NGAS demonstrations use occulters with a flight-like profile. The experiments from Princeton use an inverse occulter that could not be implemented in flight.

The throughput of the occulter is $100 \%$ for objects lying at angular positions beyond the tips of the starshade.

\section{SUMMARY}

Figures 1 though 5 plot the raw contrast attained in each experiment as a function of angular separation. Experiments conducted at the same fractional bandwidth, $\lambda / \Delta \lambda$ are grouped together in the same figure. The starshade experiments did not use filters and so received light across the full wavelength response of the CCD detectors: approximately $400-650 \mathrm{~nm}$ and thus a fractional bandwidth of about $50 \%$.

The boundaries used to delimit the coronagraph dark holes were used to calculate the mean contrast (averaged over the entire dark hole) and the extent of the field, measured in $(\lambda / D)^{2}$. This data is shown in Table 1. Other details concerning the experiments are also listed, along with an estimate of the coronagraph throughput as discussed in Section 2.

The raw contrast data listed in Table 1 is also plotted in Fig. 6 to illustrate the wavelength dependence of the contrast with each technique. It should be noted that the current monochromatic performance of the vector vortex is now better than $10^{-9}$, as reported in Ref. 11 .

\section{ACKNOWLEDGMENTS}

Work by PRL was carried out at the Jet Propulsion Laboratory, California Institute of Technology, under contract with the National Aeronautics and Space Administration. Copyright 2013. All rights reserved. 


\section{REFERENCES}

[1] Moody, D. C., Gordon, B. L., and Trauger, J. T., "Design and demonstration of hybrid Lyot coronagraph masks for improved spectral bandwidth and throughput," Proc. SPIE 7010, 7010-3P (Aug. 2008).

[2] Trauger, J., Moody, D., Gordon, B., Krist, J., and Mawet, D., "Complex apodization Lyot coronagraphy for the direct imaging of exoplanet systems: design, fabrication, and laboratory demonstration," Proc. SPIE 8442, 8442-4Q (Sept. 2012).

[3] Guyon, O., Kern, B., Belikov, R., Shaklan, S., Kuhnert, A., Give'on, A., and Martinache, F., "Phase induced amplitude apodization (PIAA) coronagraphy: recent results and future prospects," Proc. SPIE 8442, 8442-4V (Sept. 2012).

[4] Belikov, R., Pluzhnik, E., Witteborn, F. C., Greene, T. P., Lynch, D. H., Zell, P. T., Schneider, G., Guyon, O., and Tenerelli, D., "EXCEDE technology development I: first demonstrations of high contrast at $1.2 \lambda / \mathrm{D}$ for an Explorer space telescope mission," Proc. SPIE 8442, 8442-09 (Sept. 2012).

[5] Belikov, R., Bendek, E. A., Greene, T. P., Guyon, O., Lozi, J., Lynch, D. H., Newman, K. E., Pluzhnik, E., Schneider, G., Tenerelli, D., Thomas, S. J., and Witteborn, F. C., "EXCEDE technology development II: demonstration of high contrast at $1.2 \lambda /$ D and first broadband results," Proc. SPIE 8864, 8864-32 (2013).

[6] Kern, B., Guyon, O., Give'On, A., Kuhnert, A., Niessner, A., Martinache, F., Give'On, A., and Balasubramanian, K., "Laboratory demonstration of phase induced amplitude apodization (PIAA) coronagraph with better than $10^{-9}$ contrast," Proc. SPIE 8864, 8864-26 (2013).

[7] Belikov, R., Give'on, A., Kern, B., Cady, E., Carr, M., Shaklan, S., Balasubramanian, K., White, V., Echternach, P., Dickie, M., Trauger, J., Kuhnert, A., and Kasdin, N. J., "Demonstration of high contrast in $10 \%$ broadband light with the shaped pupil coronagraph," Proc. SPIE 6693, 6693-0Y (Sept. 2007).

[8] Carlotti, A., "Apodized phase mask coronagraphs for arbitrary apertures," Astron. Astrophys. 551, A10 (Mar. 2013).

[9] Lyon, R. G., Clampin, M., Petrone, P., Mallik, U., Madison, T., and Bolcar, M. R., "High contrast vacuum nuller testbed (VNT) contrast, performance, and null control," Proc. SPIE 8442, 8442-08 (Sept. 2012).

[10] Mawet, D., Serabyn, E., Moody, D., Kern, B., Niessner, A., Kuhnert, A., Shemo, D., Chipman, R., McClain, S., and Trauger, J., "Recent results of the second generation of vector vortex coronagraphs on the highcontrast imaging testbed at JPL," Proc. SPIE 8151, 8151-1D (Sept. 2011).

[11] Serabyn, E., Mawet, D., Trauger, J. T., Moody, M., Liewer, K., Krist, J. E., and Kern, B. D., "High-contrast imaging with the vortex coronagraph," Proc. SPIE 8864, 8864-32 (2013).

[12] Sirbu, D., Cady, E. J., Kasdin, N. J., Vanderbei, R. J., Lu, J., and Kao, E., "Optical verification of occulter-based high contrast imaging," Proc. SPIE 8151, 8151-14 (Sept. 2011).

[13] Samuele, R., Varshneya, R., Johnson, T. P., Johnson, A. M. F., and Glassman, T., "Progress at the starshade testbed at Northrop Grumman Aerospace Systems: comparisons with computer simulations," Proc. SPIE 7731, 7731-51 (July 2010).

[14] Glassman, T., Casement, S., Warwick, S., Amagan, O., and Donovan, J., "Achieving high-contrast ratios with a 60-cm starshade," Proc. SPIE 8864, 8864-42 (2013). 